\title{
Habilidades do cardiologista nos cuidados paliativos e a importância do reconhecimento precoce
}

\author{
Cardiologist's skills in palliative care and the importance of early recognition
}
Las habilidades del cardiólogo en cuidados paliativos y la importancia del reconocimiento temprano

Armando Hiroyuki Mori Júnior ${ }^{1 *}$, João Marcos Bemfica Barbosa Ferreira ${ }^{2}$, Simão Gonçalves Maduro $^{2}$, Marlucia do Nascimento Nobre ${ }^{1}$, Aline Brasil Aranha ${ }^{2}$, Bárbara Pires Ihara ${ }^{1}$, Angelo Bruno Pagoto ${ }^{1}$, Irineu Lopes de Alcântara Júnior ${ }^{1}$, Diego Miléo de Oliveira Freitas ${ }^{1}$.

\section{RESUMO}

Objetivo: Discutir e revisar sobre a importância do cardiologista no reconhecimento precoce e na abordagem da insuficiência cardíaca como doença cardíaca terminal. Revisão bibliográfica: As doenças cardiovasculares são a causa número um de morte global, sendo a insuficiência cardíaca o protótipo da doença cardíaca avançada. Segundo a Organização Mundial de Saúde foi incluído um grupo de doenças cardiovasculares como primeira demanda para cuidados paliativos no mundo, sendo que, a insuficiência cardíaca representa um desafio na avaliação prognóstica, uma vez que muitos pacientes morrem subitamente, mesmo em classes funcionais iniciais. Muitos desses pacientes são avaliados em um momento tardio de sua doença, e a grande maioria não é abordada pelo seu cardiologista assistente. Considerações finais: O conhecimento técnico da doença, aliado a todo o trabalho precoce com o paciente e a família podem proporcionar um tratamento otimizado e um adequado controle de sintomas em todos os momentos da doença, podendo diminuir o número de mortes precoces e aumento na expectativa de vida.

Palavras-chave: Insuficiência cardíaca, Doença cardíaca terminal, Cuidados paliativos.

\begin{abstract}
Objective: To discuss and review the importance of the cardiologist in the early recognition and approach of heart failure as terminal heart disease. Bibliographic review: Cardiovascular diseases are the number one cause of global death, with heart failure being the prototype of advanced heart disease. According to the World Health Organization, a group of cardiovascular diseases was included as the first demand for palliative care in the world, and heart failure represents a challenge in the prognostic evaluation, since many patients die suddenly, even in initial functional classes. Many of these patients are evaluated at a late stage of their illness, and the vast majority are not approached by their assistant cardiologist. Final considerations: Technical knowledge of the disease, combined with all early work with the patient and family, can provide optimized treatment and adequate symptom control at all times of the disease, which can reduce the number of early deaths and increase in expectation of life.
\end{abstract}

Key words: Heart failure, Terminal heart disease, Palliative care.

\footnotetext{
${ }^{1}$ Hospital Universitário Getúlio Vargas (HUGV), Manaus - AM. *E-mail: hiro_mori@hotmail.com

${ }^{2}$ Hospital Universitário Francisca Mendes (HUFM), Manaus - AM.
}

SUBMETIDO EM: 4/2021

ACEITO EM: 4/2021

PUBLICADO EM: 4/2021 


\section{RESUMEN}

Objetivo: Discutir y revisar la importancia del cardiólogo en el reconocimiento precoz y abordaje de la insuficiencia cardíaca como cardiopatía terminal. Revisión bibliográfica: Las enfermedades cardiovasculares son la causa número uno de muerte mundial, siendo la insuficiencia cardíaca el prototipo de la enfermedad cardíaca avanzada. Según la Organización Mundial de la Salud, se incluyó un grupo de enfermedades cardiovasculares como la primera demanda de cuidados paliativos en el mundo, y la insuficiencia cardíaca representa un desafío en la evaluación pronóstica, ya que muchos pacientes mueren repentinamente, incluso en clases funcionales iniciales. Muchos de estos pacientes son evaluados en una etapa tardía de su enfermedad y a la gran mayoría no son tratados por su cardiólogo asistente. Consideraciones finales: El conocimiento técnico de la enfermedad, combinado con todo el trabajo temprano con el paciente y la familia puede proporcionar un tratamiento optimizado y un adecuado control de los síntomas en todo momento de la enfermedad, lo que puede reducir el número de muertes tempranas y aumentar la esperanza de vida.

Palabras clave: Insuficiencia cardíaca, Enfermedad cardíaca terminal, Cuidados paliativos.

\section{INTRODUÇÃO}

O processo de mudanças na sociedade ao longo das décadas gerou diversos problemas para a população, com isso, sabe-se que cada vez mais é necessário fornecer suporte médico, familiar e emocional a esses doentes, entendendo a individualidade de cada caso e se adequando a eles (BRABO BCF e LAPRANO MGG, 2018).

Nesse cenário, tem-se que cerca de 40 milhões no mundo necessitando desses cuidados paliativos e desses cerca de $40 \%$ são advindos de doenças cardiovasculares. Os Cuidados Paliativos (CP) podem ser definidos como um método de assistência que é feito pela equipe multiprofissional, com o objetivo de melhorar a qualidade de vida do paciente e de seus familiares, quando se trata de uma doença que afete a continuidade da vida, aliviando os sintomas. Isso levando em consideração os aspectos biopsicossociais e espirituais (MATSUMOTO DY, 2012).

Tratar acerca dos CP em Cardiologia não se trata apenas de pacientes "terminais", ao contrário do que muitos pensam, trata-se de precocemente oferecer um modo diferente de lidar com doenças crônicas, favorecendo o entendimento do paciente sobre a patologia, a sua aceitação e a tomada de medidas progressivamente a fim de melhorar sua qualidade de vida e tentar regredir ou diminuir o avanço da doença (BERNOCHE C, et al., 2019).

Quanto aos CP, ainda que se trate de um problema com enorme contingente de afetados, existem muitas dificuldades de sua implementação no cotidiano. Isso se dá primariamente por certo desconhecimento acerca do assunto por parte da equipe multiprofissional e também pelos próprios pacientes. Além disso, diversos tipos de doenças cardiovasculares têm sua história natural com várias fases e cada uma delas teriam uma organização paliativa diversa. Essas variações necessitam de profissionais altamente capacitados na área de cuidados paliativos na cardiologia (PEDRÃO TGG, et al., 2018; BRABO BCF e LAPRANO MGG, 2018).

Sabe-se que, no Brasil, poucas escolas médicas têm matérias voltadas aos Cuidados Paliativos e sua importância, tendo em vista que o paciente deve ser entendido como um ser que está adoecido, mas que tem várias convicções familiares, emocionais e espirituais. Por isso, a boa prática médica atualmente exige do profissional essa visão mais humana das necessidades do doente (CALDAS GHDO, et al., 2018).

Dessa forma, o presente artigo tem como finalidade fazer uma revisão de literatura acerca do tema CP, haja vista que tal característica médica é extremamente necessária para todo e qualquer profissional da área, uma vez que nem sempre é possível curar, mas sempre é possível cuidar. Por isso esse trabalho pretende mostrar que amenizar o sofrimento do paciente é mais que simples formação acadêmica, é sim arte de se ter empatia e para isso agregamos um vasto conteúdo literário que serve como análise da importância e também da aplicabilidade do cuidado paliativo. 


\section{REVISÃO BIBLIOGRÁFICA}

\section{Epidemiologia das doenças cardiovasculares}

As doenças cardiovasculares (DCV) são a causa número um de morte global. Estima-se que 17.9 milhões de pessoas morreram de DCV em 2016, representando 31\% das mortes em todo o mundo. Dentre essas mortes, $85 \%$ são devido a infarto do miocárdio e acidente vascular encefálico (WHO, 2021).

No Brasil, a proporção de dados de 2016 mostra que as DCV são responsáveis por 27,6\% dos 1.309 .774 óbitos registrados no DATASUS, correspondendo a 33\% dos custos do Sistema Único de Saúde (SUS) (VIANA PAS, et al., 2018).

Embora as doenças cardiovasculares sejam mais comuns com a idade avançada, principalmente a insuficiência cardíaca (IC), elas não se restringem aos idosos. Fatores como pressão arterial elevada, tabagismo, altos níveis de glicose sanguínea e sedentarismo podem levar pacientes mais jovens a desenvolverem tais doenças. Tais fatores podem ser amenizados ou tratados pelas medidas dos cuidados paliativos. Além disso, sabe-se que mais de 152.000 americanos com idade inferior a 65 anos morrem de doenças cardiovasculares por ano, sendo a morte subida a causa mais característica, que poderia ser evitada com medidas voltadas ao assunto dos CP (TESTON EF, et al., 2016).

\section{Reconhecimento da doença cardíaca terminal}

A IC representa o protótipo da doença cardíaca avançada. Embora haja muitas evidências de tratamentos que auxiliem nos sinais e sintomas, assim como no aumento da sobrevida, ainda se trata de uma doença de evolução progressiva, cuja sobrecarga física e psicológica para os pacientes e seus familiares pode se estender por longos períodos de tempo (POFFO MR, et al., 2017).

Com relação apenas a IC, a OMS aponta uma prevalência de 23 milhões de casos no mundo. Nos países desenvolvidos, acomete de 1 a $2 \%$ da população adulta, com uma prevalência de até $10 \%$ dos idosos. No caso do Brasil, o DATA SUS indica cerca de 2 milhões de pacientes acometidos, com 240 mil casos diagnosticados ao ano, com estimativas de aumento do número de casos a medida que a população envelhece. (VIANA PAS, et al., 2018)

De acordo com a Organização Mundial de Saúde (OMS) (2015) houve a inclusão do grupo de doenças cardiovasculares como primeira demanda para cuidados paliativos no mundo, frente a doenças oncológicas, que configuraram na segunda posição. Apesar desse reconhecimento, o acesso a serviços de Cuidados Paliativos ainda é restrito, sendo essa falta de acesso uma realidade para o mundo todo, incluindo o Brasil.

Geralmente a tomada de decisões em pacientes cardiopatas é realizada por cardiologistas que, geralmente, não possuem formação em CP. Os paliativistas geralmente são solicitados para interconsultas apenas nos momentos finais de vida, na qual os CP só são requisitados após esgotadas as alternativas de tratamento modificador da doença. No entanto, apenas em casos raros o quadro grave ocorrerá sem um histórico prévio. A apresentação típica se dá no paciente já sabidamente portador de IC, com progressão dos sintomas ao longo do tempo (geralmente anos), possibilitando a ação efetiva dos CP na qualidade de vida do paciente, podendo aumentar sua expectativa de vida. (ORZECHOWSKI R, et al., 2019).

Apesar dos significativos avanços no tratamento da IC, permanece uma doença progressiva, extremamente sintomática e terminal. A IC não tratada caracteriza-se pela piora dos sintomas, admissões hospitalares de urgência devido a descompensações agudas, desenvolvimento de complicações (como as arritmias) e curta sobrevida (MESQUITA ET, et al., 2017).

Existem muitos sistemas de classificação de gravidade de IC, e estes vão além da simples fração de ejeção. Duas classificações muito utilizadas são: a classificação segundo a classe funcional pela New York Heart Association e segundo o estágio da doença pela American Collegy of Cardiology (Quadro 1 e Quadro 2). 
Quadro 1 - Classificação funcional, segundo a New York Heart Association (NYHA).

\begin{tabular}{|l|l|l|}
\hline Classe & Definição & Descrição Geral \\
\hline NYHA I & Ausência de sintomas & Assintomático \\
\hline NYHA II & Atividades físicas habituais causam sintomas. Limitação leve. & Sintomas leves \\
\hline NYHA III & $\begin{array}{l}\text { Atividades físicas, menos intensas que as habituais, causam } \\
\text { sintomas. Limitação importante, porém confortável no repouso. }\end{array}$ & Sintomas moderados \\
\hline NYHA IV & $\begin{array}{l}\text { Incapacidade para realizar qualquer atividade física sem apresentar } \\
\text { desconforto. Sintomas no repouso. }\end{array}$ & Sintomas graves \\
\hline
\end{tabular}

Fonte: Júnior AHM, et al., 2021. Adaptado de Dolgin M, et al., 1994.

Quadro 2 - Estágios da insuficiência cardíaca (IC), segundo American College of Cardiology / American Heart Association.

\begin{tabular}{|c|l|}
\hline Estágio & Descrição \\
\hline A & Risco de desenvolver IC. Sem doença estrutural ou sintomas de IC \\
\hline B & Doença estrutural cardíaco presente. Sem sintomas de IC \\
\hline C & Doença estrutural cardíaco presente. Sintomas prévios ou atuais de IC \\
\hline D & IC refratária ao tratamento clínico. Requer intervenção especializada \\
\hline
\end{tabular}

Fonte: Júnior AHM, et al., 2021. Adaptado de Bocchi EA, 2009

Além das definições americanas quanto às doenças cardiovasculares, tem-se diversos outros critérios de classificação, como o da Sociedade Europeia de Cardiologia, que leva em conta os diversos desdobramentos da IC avançada, sendo que ela pode ser definida pela presença de um ou mais dos critérios elencados (Quadro 3).

Quadro 3 - Critérios da Sociedade Europeia de Cardiologia para a definição de Insuficiência Cardíaca Avançada.

\begin{tabular}{|c|c|}
\hline ério & escrição \\
\hline I & $\begin{array}{l}\text { ntomas graves de IC com dispneia e/ou fadiga em repouso ou aos mínimos esforções (CF III } \\
\text { I IV da NYHA). }\end{array}$ \\
\hline II & $\begin{array}{l}\text { Episódios de retenção hídrica (congestão sistêmica ou pulmonar e/ou edema periférico) e/ou } \\
\text { redução do débito cardíaco em repouso (hipoperfusão periférica). }\end{array}$ \\
\hline III & $\begin{array}{l}\text { Evidência objetiva de disfunção cardíaca grave, demonstrando através de pelo menos um dos } \\
\text { seguintes itens: } \\
\text { a) Uma baixa Fração de ejeção de Ventrículo Esquerdo (VE) }(<30 \%) \text {; } \\
\text { b) Uma anormalidade grave da função cardíaca ao ecocardiograma Doppler com padrão } \\
\text { de fluxo mitral restritivo ou pseudonormal; } \\
\text { c) Altas pressões de enchimento de VE [Pressão de capilar pulmonar (PCP) média > } \\
16 \mathrm{mmHg} \text {, e/ou Pressão de átrio direito (PAD) média > 12mmHg por cateterização de } \\
\text { artéria pulmonar]; } \\
\text { Níveis plasmáticos elevados de BNP ou NT-proBNP, na ausência de causas não cardíacas. }\end{array}$ \\
\hline IV & $\begin{array}{l}\text { Prejuízo grave da capacidade funcional, demonstrado por um dos seguintes itens: } \\
\text { a) Inabilidade para o exercício; } \\
\text { b) Distância < } 300 \mathrm{~m} \text { no teste de caminhada de } 6 \text { minutos ou menos em mulheres e/ou } \\
\text { pacientes com idade >= } 75 \text { anos; } \\
\text { Vo2 pico < } 12 \text { a } 14 \mathrm{ml} / \mathrm{kg} / \mathrm{min} \text {. }\end{array}$ \\
\hline V & História de uma ou mais hospitalizações por IC nos últimos 6 meses. \\
\hline VI & $\begin{array}{l}\text { Presença de todas as características prévias com terapia otimizada, incluindo diuréticos, } \\
\text { inibidores do sistema renina-angiotensina-aldosterona e beta-bloqueadores, ao menos que } \\
\text { esta seja mal tolerada ou contraindicada, e Terapia de ressincronização cardíaca, quando } \\
\text { indicada. }\end{array}$ \\
\hline
\end{tabular}

Fonte: Júnior AHM, et al., 2021. Adaptado de Ponikowski P, et al., 2016. 
Do ponto de vista teórico, a OMS instituiu o conceito de CP pela primeira vez em 1990, e em 2002 o revisou e o ampliou para uma condição que envolve todos os pacientes portadores de doenças graves, incuráveis e progressivas, que ameacem a continuidade da vida. A exemplo de acometimentos como a aids, doenças neurológicas, e, principalmente, doenças cardiovasculares (HERMES HR, et al., 2013).

No entanto, se esta conceituação fosse assumida de maneira literal, o número de pacientes acometidos e com indicação de CP seria consideravelmente expressivo e não haveria como prestar este tipo de assistência a todos. Por este motivo, a Academia Nacional de Cuidados Paliativos (ANCP) (2009) recomendou a adoção de critérios que avaliassem e permitissem uma melhor prestação dos cuidados ao doente. Dentre os quais, abrange-se o diagnóstico de uma doença incurável, a expectativa de vida inferior a 6 meses, a disponibilidade operacional para implementação dos cuidados e a opção do paciente pela realização dos CP em detrimento da continuidade de seu tratamento.

Dessa maneira, além das condições do portador de IC, que em determinadas situações apresenta um quadro de incerteza em seu prognóstico e em sua fase final de vida, pacientes com indicação para transplante cardiovascular, bem como transplante de outros órgãos sólidos, são candidatos para os $\mathrm{CP}$, em virtude da probabilidade elevada de determinar um grande sofrimento em diversos casos, gerando um quanto sintomático de grande intensidade e desconforto (TAVARES CR, et al., 2013).

\section{Sintomas prevalentes}

É evidente que os avanços terapêuticos vêm garantindo uma melhora no tratamento e no prognóstico de pacientes cardiopatas, assegurando uma melhor sobrevida aos pacientes com IC. No entanto, essa melhora ainda não é capaz de evitar a progressão da doença, que cursa com uma sintomatologia incapacitante, seja ela física ou emocional, gerando ao paciente uma qualidade de vida gravemente comprometida. Quanto mais grave e avançado é o acometimento, menor é a disponibilidade de intervenções que prolonguem a vida e maior é a necessidade de ações de cuidados paliativos (AFONSO BQ, et al., 2020).

Durante a evolução natural da doença o paciente cardiopata apresenta sintomas que decorrem tanto das alterações anatômico funcionais cardíacas como do impacto do próprio tratamento em si. Os sintomas físicos são os maiores preditores de perda da qualidade de vida, dentre eles, a fadiga é considerada um dos mais frequentes nos pacientes com IC, sendo referenciada na literatura com valores de acometimento desses pacientes em até $85 \%$ dos casos. Em conjunto da dispneia e do edema, forma a tríade clínica clássica em pacientes idosos que cursam com IC (BORGES JA, et al., 2018).

A experiência dos sintomas de cada paciente é individual e subjetiva, devendo a equipe assistencial multiprofissional usar o próprio paciente como referência para interpretação e melhor abordagem. Desse modo, as intervenções que melhoram a qualidade de saúde do paciente têm impacto direto na mortalidade, eventos clínicos, necessidade de internações e custos (RUMSFELD JS, et al., 2013).

\section{Dispneia}

A dispneia é uma experiência subjetiva de desconforto respiratório, resultante de aspectos fisiológicos, psicológicos, sociais e ambientais e em pacientes com cardiopatia é um dos sintomas mais relevantes (CHERNY NI, et al., 2015).

É o sintoma mais frequente no momento da admissão no pronto-socorro de pacientes com IC, sendo considerado também como um dos mais intensos e relacionado com a piora do desempenho funcional do acometido. De tal maneira que contribui para o efeito debilitante da doença, favorecendo ao isolamento social e o risco aumentado de descompensação da IC (KUROGI EM, et al., 2020).

No cardiopata a congestão pulmonar é muito frequente, mas não é a única causa da dispneia para sua gênese. Hipertensão pulmonar, derrame pleural, descondicionamento e fraqueza da musculatura respiratória, doenças pulmonares associadas, causas restritivas, aspectos não orgânicos, entre outros, podem ser responsáveis pela dispneia. Portanto, o tratamento da dispneia parte do princípio da necessidade de identificação da causa e seu tratamento específico (PARSHALL MB, et al., 2012). 


\section{Fadiga}

A fadiga acomete em torno de $30 \%$ a $60 \%$ dos pacientes com IC e até $80 \%$ apresentam fadiga e dispneia, sendo $20 \%$ com sintomas em repouso. Entretanto, muitas vezes é subdiagnosticada e subtratada, estando associada a pior qualidade de vida, prognóstico e mortalidade (HEO S, et al., 2008; PEREZ-MORENO AC, et al., 2014).

Essa dificuldade no diagnóstico deve-se principalmente ao fato de a fadiga não possuir um conceito claro e bem definido na prática clínica diária. Muitos sinônimos são associados à fadiga de maneira equivocada, porém, muitos destes são sintomas associados que cursam em conjunto a fadiga como astenia, letargia, cansaço extremo, sensação de fraqueza, entre outros. O conceito mais utilizado para o termo na literatura científica é o que denota a fadiga como sendo uma sensação subjetiva de exaustão desproporcional à atividade que vem sendo exercida (BORGES JA, et al., 2018).

Quando o quadro sintomático não apresenta melhora dessa sensação por um período superior a seis meses, mesmo quando o paciente está em repouso, tem se a caracterização da Síndrome da Fadiga Crônica (SFC), que é marcada por um quadro debilitante, geralmente com etiologia específica desconhecida e sem tratamento curativo (VIEIRA CMS, et al., 2020).

Desse modo, é necessário investigar causas secundárias responsáveis pela fadiga que possa ser corrigida com tratamento específico, podendo ser em razão do descondicionamento físico, perda de massa muscular, distúrbios do sono, depressão e/ou ansiedade, condições metabólicas (por exemplo, anemia e hipotireoidismo, hiponatremia e hipocalemia, infecções), entre outros. No paciente com IC o próprio baixo débito pode ser o responsável pela fadiga (BORGES JA, et al., 2018).

Em fases mais precoces é possível um trabalho voltado para reabilitação, condicionamento físico e incentivo ao exercício, permitindo melhor tolerância aos esforços. Já em fase final de vida, prefere-se medidas para poupar energia e garantir uma melhora na qualidade de vida do acometido (EVANGELISTA LS, et al., 2008).

\section{Edema}

O edema é um dos sintomas que mais afeta os pacientes cardiopatas, acometendo principalmente os membros inferiores. Com a evolução da doença, restrição ao leito, caquexia, hipoalbuminemia, piora da função renal e perda da capacidade de eliminar uma diurese volumosa, o edema progride para dorso, sacro e até membros superiores, chegando a transudar pela pele em casos mais graves (CLARK AL, et al., 2013).

O edema, especialmente em pacientes que possuem IC, prejudica o ventrículo em ejetar o sangue, levando a congestão pulmonar e ao edema periférico. Pacientes com edema têm maior risco de complicações como lesões de pele (lesões por pressão) e infecções (celulite), que podem ser causas de descompensação da IC. Tais características de congestão são apontadas como importantes fatores de hospitalização e prognóstico de pacientes cardiopatas. De tal maneira, a atenção multidisciplinar é fundamental, bem como a orientação dietética (restrição hidrossalina), os cuidados com a pele, exercícios, diferentes posicionamentos, acolhimento e aconselhamento familiar (COSTA MB, et al., 2019).

É importante salientar que o ganho de peso diário no paciente cardiopata reflete o acúmulo de líquidos. Uma família vigilante e orientada pode ser empoderada ao reconhecer tal condição e quanto a necessidade de reforçar a restrição hídrica e dispor de doses adicionais de diurético com o objetivo de retornar ao peso ideal estipulado (RICARDO TC, et al., 2019).

\section{Sintomas do trato gastrointestinal}

Pacientes com IC frequentemente apresentam sintomas gastrointestinais como náuseas, constipação e dor abdominal. O baixo débito cardíaco associado a congestão hepática e de alças intestinais são responsáveis pela gênese de maior parte dos sintomas. As náuseas podem ser em razão da redução no esvaziamento gástrico, doença péptica gástrica, falência hepática ou renal e uso de medicamentos, como a Digoxina. Já a constipação pode ser secundária a congestão de alças intestinais e imobilismo (CHERNY NI, et al., 2015). 
No caso específico do uso de medicações para o tratamento da IC, a Digoxina apresenta resultados satisfatórios na redução das hospitalizações dos pacientes, no entanto, apresenta como um de seus principais efeitos colaterais os sintomas gastrointestinais, principalmente o vômito, a anorexia e a náusea (FREITAS AKE e CIRINO RHD, 2017).

\section{Dor}

A dor é um sintoma muitas vezes subdiagnosticado ou pouco valorizado na prática clínica em pacientes com IC, quando as atenções são voltadas para sintomas mais exuberantes, como dispneia. A prevalência pode variar de $20 \%$ a $84 \%$, todavia muitos pacientes apresentam mais de um sítio de dor simultaneamente (GOODLIN SJ, et al., 2010).

As mais frequentes referidas são as dores osteomusculares em membros inferiores e costas, sendo a artrite muito comum na população mais idosa. Também há queixas frequentes de dores torácicas, relacionadas ou não com fenômenos anginosos. É vital reconhecer a dor e o desconforto relacionados com choques de desfibriladores implantáveis e também dos componentes da "dor total" devendo-se avaliar não apenas físicos causadores de dor, mas aspectos psicológicos, sociais e espirituais que colaboram como gênese da dor (CHERNY N, et al., 2015).

\section{Depressão}

Sintomas psicológicos, associados a perda do bem-estar espiritual e social, permeiam a evolução da cardiopatia e têm influência sobre sintomas físicos e afetam diretamente a qualidade de vida, funcionalidade, prognóstico e custos com saúde. A depressão, especificamente, tem uma prevalência estimada entre $20 \%$ e $40 \%$ e é associada a piores desfechos nessa população (FARIS R, et al., 2002).

Quanto mais grave é a depressão, piores são os sintomas físicos, independentemente de sinais clínicos ou radiológicos objetivos de gravidade da cardiopatia. Possui um impacto negativo principalmente sobre a fadiga, dispneia e dor torácica (SULLIVAN M, et al., 2004).

Isso deve-se ao fato de ocorrerem inúmeras alteração fisiometabólicas no organismo de pacientes que cursam com a depressão associada a IC, como disglicemia, hipercolesterolemia, decréscimo da variabilidade da frequência cardíaca, níveis séricos elevados de citocinas pró-inflamatórias, entre outras. Pacientes com depressão também possuem maior risco de não aderência medicamentosa e hábitos de vida inadequados, predispondo a descompensação clínica, necessidade de internações e pior prognóstico (GUERRA TRB e MESQUISA ET, 2020; FREEDLAND KE, et al., 2015).

\section{Fatores prognósticos}

Das cardiopatias, a IC é a que representa alguns dos maiores estorvos, principalmente no tocante a avaliação prognóstica. Isso porque muitos pacientes morrem subitamente, mesmo em classes funcionais iniciais. Uma parcela majoritária compartilha caminho de declínio gradual e contínuo, que normalmente dura alguns anos, associado a episódios de deteriorações agudas, por vezes reversíveis, mas que também podem levar a morte (FEITOSA-FILHO GS, et al., 2019).

Todo esse percurso pode ser dividido em três fases: a primeira, relativamente estável, momento em que ocorre o manejo da rotina relacionado com a doença crônica; a segunda, em que o uso de cuidados hospitalares se torna mais frequente à medida que há um visível declínio de funcionalidade, com uma variedade de estratégias de cuidados de suporte e paliativos sendo implementados; e a terceira, momento em que ocorre uma deterioração inexorável, período que pode durar dias a semanas (GADOUD A, et al., 2013).

A cada internação hospitalar acumulada, a sobrevida mediana diminui. Após a quarta internação hospitalar decorrente de questões cardíacas, metade dos pacientes morrem após um período de 7 meses (SETOGUCHI S, et al., 2007).

No Reino Unido, recomenda-se o uso de uma ferramenta específica que auxilia na detecção de pacientes em seus últimos 12 meses de vida, incluindo pacientes portadores de cardiopatias: o guia de indicador 
prognóstico Gold Standards Framework (GSF). São três as etapas chaves do processo: identificação de pacientes; avaliação das necessidades clínicas e pessoais; planejamento do cuidado (HAGA K, et al., 2012).

Quadro 5 - Passos para o processo de identificação de paciente em seus últimos 12 meses de vida.

\begin{tabular}{|c|c|c|}
\hline \multicolumn{3}{|l|}{ Passo 1 - Pergunta surpresa } \\
\hline \multicolumn{3}{|c|}{ "Você se surpreenderia se este paciente morresse no próximo ano, meses, semanas ou dias?" } \\
\hline Não & Não Sei & Sim \\
\hline $\begin{array}{l}\text { Iniciar o Processo de GSF } \\
\text { (Identificar - Avaliar - } \\
\text { Planejar) }\end{array}$ & Próximo passo & Reavaliar regularmente \\
\hline \multicolumn{3}{|c|}{ Passo 2 - O paciente possui algum dos indicadores gerais de declínio e maior dependência? } \\
\hline \multicolumn{3}{|c|}{$\begin{array}{l}\text { Declínio físico geral, aumento da dependência e maior necessidade de auxílio } \\
\text { Admissões hospitalares não planejadas de repetição } \\
\text { Doença avançada - instável, em deterioração, com carga de sintomas complexos } \\
\text { Presença de multimorbidades } \\
\text { Diminuição das atividades - queda de funcionalidade limitada ao autocuidado, acamado ou sentado por } \\
\text { mais de } 50 \% \text { do tempo e aumento de dependência na maioria das atividades de vida diária } \\
\text { Diminuição de resposta a tratamentos, diminuição da possibilidade de reversão do quadro } \\
\text { Escolha do paciente de não realizar mais tratamento ativo e priorizar qualidade de vida } \\
\text { Perda ponderal progressiva (>10\%) nos últimos seis meses } \\
\text {. Evento sentinela (por exemplo, quedas graves, transferência para instituições de longa permanência) } \\
\text { Albumina sérica }<2,5 \mathrm{mg} / \mathrm{dL}\end{array}$} \\
\hline Sim & Não Sei & Não \\
\hline $\begin{array}{c}\text { Iniciar o Processo de GSF } \\
\text { (Identificar - Avaliar - } \\
\text { Planejar) }\end{array}$ & Próximo passo & Reavaliar regularmente \\
\hline \multicolumn{3}{|c|}{ Passo 3 - O paciente apresenta algum dos Indicadores clínicos específicos para IC? } \\
\hline \multicolumn{3}{|c|}{$\begin{array}{l}\text { Doença Cardíaca } \\
\text { Pelo menos dois dos indicadores abaixo: } \\
\text { Paciente para o qual a pergunta surpresa é aplicável } \\
\text {. NYHA estágio III ou IV com progressão de sintomas apesar da terapia otimizada para IC } \\
\text {. Internações de repetição por IC descompensada }-3 \text { admissões em } 6 \text { meses ou uma única internação se } \\
>75 \text { anos ( } 50 \% \text { de mortalidade em } 1 \text { ano) } \\
\text { Sintomas físicos ou psíquicos de difícil controle apesar da terapia otimizada } \\
\text {. Fatores adicionais incluindo hiponatremia }<135 \mathrm{mmol} / \mathrm{L} \text {, declínio da função renal, anemia, etc. }\end{array}$} \\
\hline \multicolumn{2}{|l|}{ Sim } & Não \\
\hline $\begin{array}{l}\text { Iniciar o Processo d } \\
\text { (Identificar - Avaliar - }\end{array}$ & & liar regularmente \\
\hline
\end{tabular}

Fonte: Júnior AHM, et al., 2021. Adaptado de Ricardo TC, et al., 2016.

O GSF foi submetido a uma avaliação por um grupo de pesquisadores quanto a sua utilidade. $O$ trabalho evidenciou uma sensibilidade de $83 \%$ e uma especificidade de $22 \%$. Se o objetivo principal for a diminuição das taxas de não reconhecimento do paciente em seu último ano de vida, a ferramenta se torna válida, embora a custa de falso positivo devido sua avaliação intuitiva (HAGA K, et al., 2012).

O desafio de dar o prognóstico de um paciente com Insuficiência Cardíaca faz com que seja difícil discutir com ele sobre as preferências de cuidado que ele deseja. Por isso, os pacientes com IC possuem pouquíssima noção de sua verdadeira condição clínica e como consequência possuem pouco comprometimento na decisão de como vai se proceder os seus cuidados (FEITOSA-FILHO GS, et al., 2019). 


\section{Cuidados paliativos na insuficiência cardíaca}

O papel numa fase mais precoce da doença muito provavelmente será realizado por profissionais sem experiência em CP. Portanto, faz-se necessário a inserção de ensino sobre conceitos básicos de CP (principalmente desmitificando como sinônimo de proximidade da morte), controle básico de sintomas, técnicas de comunicação e medicina centrada no paciente na formação do novo cardiologista. Sabe-se que, de acordo com estudo realizado em 2017, a otimização dos tratamentos cardiológicos gerou uma diminuição sensível no número de mortes precoces e consequente aumento na expectativa de vida (WARRAICH HJ, et al., 2017).

Outro levantamento realizado em 2019 demonstrou o perfil epidemiológico dos pacientes que foram encaminhados para serviços de CP na área da cardiologia. Tal estudo evidenciou que geralmente só se leva em consideração a utilização dos cuidados paliativos em pacientes de casos avançados, sendo que cerca de $33 \%$ estavam acamados e com sintomas descompensados. Além disso, fato curioso é que o encaminhamento geralmente era feito pelo médico clínico e não pelo cardiologista assistente do paciente (WARRAICH HJ, et al., 2019).

O curso da doença dificilmente será linear na sintomática. O conhecimento técnico da doença, aliado a todo o trabalho precoce com o paciente e a família podem proporcionar o entendimento real em relação a gravidade do quadro, tanto para a possibilidade do óbito, quanto para a possibilidade de se alcançar uma estabilidade, mesmo que temporária, até uma nova descompensação. Embora o falecimento ainda não tenha ocorrido, a perda da pessoa amada pode se manifestar no não reconhecimento do paciente que se encontra no leito como sujeito que foi em vida, ou ainda no início da sedação paliativa, momento em que a vida orgânica se mantém, porém, a vida biográfica se acaba (RICARDO TC, et al., 2019).

\section{CONSIDERAÇÕES FINAIS}

É de suma importância que os cardiologistas saibam acerca da CP e estratificar os pacientes que podem se beneficiar desse tratamento, fazendo com que haja uma terapêutica individualizada e com maior eficácia. Os cardiologistas devem estar preparados para uma grande variedade de desafios referentes ao tema. Eles têm de lidar regularmente com pacientes e famílias para tomarem decisões quando efetuarem um tratamento agressivo (ao serem confrontados com condições de elevada mortalidade), quando interromperem o suporte e quando iniciarem as ordens de não ressuscitação.

\section{REFERÊNCIAS}

1. $\mathrm{AFONSO} B Q$, et al. Validação do resultado controle dos sintomas para pacientes com insuficiência cardíaca em cuidados paliativos. Rev Gaucha Enferm, 2020; 41(1): e20190427.

2. BERNOCHE C, et al. Atualização da diretriz de ressuscitação cardiopulmonar e cuidados cardiovasculares de emergência da Sociedade Brasileira de Cardiologia-2019. Arquivos brasileiros de cardiologia, 2019; 113(3).

3. BORGES JA, et al. Fadiga: Um Sintoma Complexo e seu Impacto no Câncer e na Insuficiência Cardíaca. Int. J. Cardiovasc. Sci, 2018; 31(4): 433-42.

4. BORGES JA, et al. Fadiga: Um Sintoma Complexo e seu Impacto no Câncer e na Insuficiência Cardíaca. Int. J. Cardiovasc. Sci, 2018; 31(4): 433-42.

5. BRABO BCF, LAPRANO MGG. Competências do enfermeiro para o cuidado paliativo em cardiologia. Rev. enferm. UFPE online, 2018; 2341-2348.

6. BRASIL. Academia Nacional De Cuidados Paliativos (ANCP). Manual de Cuidados Paliativos ANCP. 2009. Disponível em: http://biblioteca.cofen.gov.br/wp-content/uploads/2017/05/Manual-de-cuidados-paliativos-ANCP. Acessado em: 26 de janeiro de 2021.

7. CALDAS GHDO, et al. Cuidados paliativos: uma proposta para o ensino da graduação em Medicina. Revista Brasileira de Geriatria e Gerontologia, 2018; 21 (3): 261-271.

8. CHERNY NI, et al. Oxford's Textbook of palliative medicine. Oxford University Press, USA, 2015; 5th edition.

9. CLARK AL, CLELAND JGF. Causes and treatment of oedema in patients with heart failure. Nature Reviews Cardiology, 2013; $10(3): 156$.

10. COSTA MB, et al. Intervenções de enfermagem para redução do edema de pacientes com insuficiência cardíaca hospitalizados. Nursing: Enfermagem, 2019; 250(22): 2745-2750. 
11. EVANGELISTA LS, et al. Correlates of fatigue in patients with heart failure. Progress in Cardiovascular nursing, 2008; $23(1): 12-17$.

12. FARIAS R, et al. Clinical depression is common and significantly associated with reduced survival in patients with non ischaemic heart failure. European Journal of Heart Failure, 2002; 4 (4): 541-551.

13. FEITOSA-FILHO GS, et al. Updated Geriatric Cardiology Guidelines of the Brazilian Society of Cardiology2019. Arquivos brasileiros de cardiologia, 2019; 112 (5): 649-705.

14. FREEDLAND KE, et al. Cognitive Behavior therapy for depression and self care in heart failure patients: a randomized clinical trial. JAMA Internal Medicine, 2015; 175 (11): 1773-1782.

15. FREITAS AKE, CIRINO RHD. Manejo ambulatorial da Insuficiência Cardíaca Crônica. Revista Médica da Ufpr, 2017; 3(4): 123-126.

16. GADOUD A, et al. Palliative care for people with heart failure: summary of current evidence and future direction. Palliative Medicine, 2013; 27 (9): 822-828.

17. GUERRA TRB, et al. Visão metabolômica envolvendo depressão e insuficiência cardíaca: uma análise reflexiva. Research, Society And Development, 2020; 9(8): 1-10.

18. HAGA K, et al. Identifying community based chronic heart failure patients in the last year of life: a comparison of the Gold Standards Framework Prognostic Indicator Guide and the Seattle Heart Failure Model. Heart, 2012; 98 (7): 579 583.

19. HEO S, et al. Predictors and effect of physical sympton status on health-related quality of life in patients with heart failure. American Journal of Critical Care, 2008; 17 (2): 124-132.

20. HERMES HR, et al. Cuidados paliativos: uma abordagem a partir das categorias profissionais de saúde. Ciência \& Saúde Coletiva, 2013; 18(9): 2577-2588.

21. KUROGI EM, et al. Relação entre capacidade funcional, desempenho e sintomas em pacientes internados com insuficiência cardíaca. Rev. Bras. Enferm, 2020; 73(4): e20190123.

22. MATSUMOTO DY. Cuidados paliativos: conceitos, fundamentos e princípios. Manual de cuidados paliativos ANCP, 2012; 2 (2): 23-24.

23. MESQUITA ET, et al. Entendendo a hospitalização em pacientes com insuficiência cardíaca. International Journal of Cardiovascular Sciences, 2017; 30 (1): 81-90.

24. ORZECHOWSKI R, et al. Necessidade de cuidados paliativos em pacientes com insuficiência cardíaca avançada internados em um hospital terciário. Revista da Escola de Enfermagem da USP, 2019; 53 (1): e03413.

25. PARSHALL MB, et al. An official American Thoracic Society statement: uptodate on the mechanisms, assessment, and mangement of dyspnea. American Journal of Respiratory and Critical Care Medicine, $2012 ; 185$ (4): 435-452.

26. PEDRÃO TGG, et al. Diagnósticos e intervenções de enfermagem para pacientes cardiológicos em cuidados paliativos. Rev. enferm. UFPE on line, 2018; 12(11):3038-3045.

27. PEREZ-MORENO AC, et al. Fatigue as a predictor of outcome in patients with failure analysis of CORONA (controlled rosuvastatin multinational trial in heart failure). Jam Coll Cardiol Heart Failure, 2014; 2 (2): 187-197.

28. POFFO MR, et al. Perfil dos pacientes internados por insuficiência cardíaca em hospital terciário. International Journal of Cardiovascular Sciences, 2017; 30 (3): 189-198.

29. RICARDO TC, et al. Cuidados paliativos: falências orgânicas. 1. Ed. Rio de janeiro: Atheneu, 2019.

30. RUMSFELD JS, et al. Cardiovascular health: the importance of measuring patient-reported health status: a cientific statement fron American Heart Association. Circulation, 2013; 127 (22): 2233-2249.

31. SETOGUCHI S, et al. Repeated hospitalizations predict mortality in the community population with heart failure. American heart journal, 2007; 154 (2): 260-266.

32. SULLIVAN M, et al. Depression and health status in patients with advance heart failure: a prospective study in tertiary care. Journal of Cardiac Failure, 2004;10 (5): 390-396.

33. TESTON EF, et al. Fatores associados às doenças cardiovasculares em adultos. Medicina (Ribeirão Preto). $2016 ; 49$ (2): 95-102.

34. VIANA PAS, et al. Perfil de pacientes internados para tratamento de insuficiência cardíaca descompensada. SANARE-Revista de Políticas Públicas, 2018; 17 (1): 15-23.

35. VIEIRA CMS, et al. Fadiga crônica e sua relação com as atividades da vida diária em população coberta pela estratégia saúde da família. Brazilian Journal Of Development, 2020; 6(5): 27193-27205.

36. WARRAICH HJ, et al. Characteristics and Trends Among Patients with Cardiovascular Disease Referred to Palliative Care. JAMA Network Open, 2019; 2 (5): 1-10.

37. WARRAICH HJ, et al. How medicine has changed the end of life for patients with cardiovascular disease. Journal of the American College of Cardiology, 2017; 70 (10): 1276-1289.

38. WORLD HEALTH ORGANIZATION (WHO). Cardiovascular disease. Disponível em: https://www.who.int/newsroom/fact-sheets/detail/cardiovascular-diseases-(cvds). Acesso em: 29 jan 2021. 